TRANSACTIONS OF THE

AMERICAN MATHEMATICAL SOCIETY

Volume 355, Number 6, Pages 2327-2340

S 0002-9947(03)03255-0

Article electronically published on January 27, 2003

\title{
AN ELEMENTARY INVARIANT PROBLEM AND GENERAL LINEAR GROUP COHOMOLOGY RESTRICTED TO THE DIAGONAL SUBGROUP
}

\author{
MARIAN F. ANTON
}

\begin{abstract}
Conjecturally, for $p$ an odd prime and $R$ a certain ring of $p$ integers, the stable general linear group $G L(R)$ and the étale model for its classifying space have isomorphic mod $p$ cohomology rings. In particular, these two cohomology rings should have the same image with respect to the restriction map to the diagonal subgroup. We show that a strong unstable version of this last property holds for any rank if $p$ is regular and certain homology classes for $S L_{2}(R)$ vanish. We check that this criterion is satisfied for $p=3$ as evidence for the conjecture.
\end{abstract}

\section{INTRODUCTION}

Let $p$ be a prime and $R=\mathbb{Z}[\zeta, 1 / p]$ the ring of $p$-integers containing a primitive $p$-th root of unity $\zeta$. A great deal of interest has been generated by the mod $p$ cohomology of the stable general general linear group $G L(R)$. Dwyer and Friedlander [4] constructed a topological space $X(R)$ based on the étale topological type of the scheme $\operatorname{Spec}(R)$ and a continuous map

$$
f: B G L(R) \rightarrow X(R)
$$

where $B G L(R)$ is the classifying space of the discrete group $G L(R)$. They showed that $f$ is injective on mod $p$ cohomology if $p$ is regular [5, 6.4]. Voevodsky [9] proved a conjecture of Milnor having as consequence the fact that $f$ is a $p$-equivalence (i.e., induces an isomorphism on $\bmod p$ cohomology) for $p=2$. Quillen [8] and Lichtenbaum [6] have studied a conjectural relationship between the algebraic $K$ theory of $R$ and the values of an associated zeta function. Their work suggests that $f$ should be a $p$-equivalence for any prime $p$.

In particular, let $D(R)$ be the subgroup of diagonal matrices inside $G L(R)$ and consider the following diagram on mod $p$ cohomology $H^{*}=H^{*}\left(-, \mathbb{F}_{p}\right)$ :

$$
H^{*} X(R) \stackrel{f^{*}}{\longrightarrow} H^{*} B G L(R) \stackrel{\text { res }}{\longrightarrow} H^{*} B D(R)
$$

where $f^{*}$ is induced by $f$ and res by the inclusion $D(R) \subset G L(R)$. If $f$ is a $p$-equivalence, then res and res $\circ f^{*}$ have the same image (see also Remark 4.11).

In this article we show that a strong unstable version of the last property holds under certain conditions. Namely, let $n$ be a nonnegative integer and $G L_{n}(R)$ the

Received by the editors May 1, 2002 and, in revised form, November 14, 2002.

2000 Mathematics Subject Classification. Primary 57T10, 20J05; Secondary 19D06, 55R40.

Key words and phrases. Etale model, linear group, cohomology, invariants. 
general linear group of rank $n$ over $R$. Then we can replace $G L, D, X, f$, res above respectively by $G L_{n}, D_{n}, X_{n}, f_{n}, r e s_{n}$ and keep everything else unchanged.

Theorem 1.1. Let $p$ be an odd regular prime, $M_{n}$ the image of $r e s_{n} \circ f_{n}^{*}$, and $I_{n}$ the image of res $s_{n}$. If $M_{2}=I_{2}$, then $M_{n}=I_{n}$ for all $n \geq 0$.

We can actually strengthen this theorem by weakening the condition $M_{2}=I_{2}$ and requiring instead that certain homology classes for $S L_{2}(R)$ vanish (see Theorem 4.10). As an example, when $M_{2}=I_{2}$ holds we can cite our previous result (see also Remark 4.4).

Corollary $1.2([1,1.3])$. If $p=3$, then $M_{n}=I_{n}$ for all $n \geq 0$.

Theorem 1.1] was inspired by [3] where a similar statement was proven in case $p=2$. Our generalization is based on two observations: (i) the mod $p$ cohomology ring of the étale model $X_{n}(R)$ has a particularly simple structure for $p$ odd and regular [5] and (ii) the maps dual to res $_{n}$ assemble for $n \geq 0$ into a ring homomorphism on homology with respect to the ring structure induced by matrix block-multiplication $G L_{n}(R) \times G L_{m}(R) \rightarrow G L_{n+m}(R)$. Based on this last ring structure, we formulate and solve a general invariant problem (Theorem 3.4) having as consequence Theorem 1.1 Surprisingly, the solution to the invariant problem is elementary and purely combinatorial, although quite tricky.

The article is organized as follows. In $₫ 2$ we review the mod $p$ cohomology ring of the étale model $X_{n}(R)$. In $\S 3$ we formulate and solve the invariant problem and deduce Theorem 1.1 In $\$ 4$ we strengthen and generalize our theorem.

We acknowledge the hospitality of the Max-Planck Institut für Mathematik in Bonn during the preparation of this article and thank an anonymous referee for accuracy and presentation suggestions.

\section{Etale model COHOMOLOGY}

In what follows we assume that $p$ is an odd regular prime and keep the notation from the Introduction. In [5, 6.3] the graded ring associated to a filtration of $H^{*} X_{n}(R)$ was computed and we review this calculation here from a slightly different perspective:

Theorem 2.1. Let $p$ be an odd regular prime and $n$ a nonnegative integer. Then

$$
H^{*} X_{n}(R) \approx \mathbb{F}_{p}\left[c_{1}, c_{2}, \ldots, c_{n}\right] \otimes \bigotimes_{i=1}^{r} \Lambda\left(e_{i, 1}, e_{i, 2}, \ldots, e_{i, n}\right)
$$

where $r=(p+1) / 2, \otimes$ is the tensor product over $\mathbb{F}_{p}$, and $\Lambda$ is the symbol for an exterior $\mathbb{F}_{p}$-algebra. For $1 \leq j \leq n$ and $1 \leq i \leq r, c_{j}$ is the Chern class of degree $\left|c_{j}\right|=2 j$ associated with a complex embedding of $R$ and $e_{i, j}$ is a class of degree $\left|e_{i, j}\right|=2 j-1$.

The following corollary is essentially [5] 6.2] in a form more suitable for the purpose of this article.

Corollary 2.2. With the same notation and hypotheses as in Theorem 2.1.

$$
H^{*} B D_{n}(R) \approx \mathbb{F}_{p}\left[x_{1}, x_{2}, \ldots, x_{n}\right] \otimes \bigotimes_{i=1}^{r} \Lambda\left(y_{i, 1}, y_{i, 2}, \ldots, y_{i, n}\right)
$$


where the degrees of the generators are given by $\left|x_{j}\right|=2$ and $\left|y_{i, j}\right|=1$ and the map res $_{n} \circ f_{n}^{*}$ is a ring monomorphism sending $c_{j}$ to the symmetrization of $x_{1} \ldots x_{j}$ and $e_{i, j}$ to the symmetrization of $x_{1} \ldots x_{j-1} y_{i, j}$ for $1 \leq j \leq n$ and $1 \leq i \leq r$.

Remark 2.3. For $n \geq 0$, let $\Sigma_{n}$ be the symmetric group of $\{1,2, \ldots, n\}$. Then $\Sigma_{n}$ acts in an obvious manner on the diagonal subgroup $D_{n}(R)$. This action induces an action of $\Sigma_{n}$ on $H^{*} D_{n}(R)$ via ring homomorphims $x \mapsto x^{\sigma}$ sending $x_{j}$ to $x_{\sigma(j)}$ and $y_{i, j}$ to $y_{i, \sigma(j)}$ for $1 \leq i \leq r$ and $1 \leq j \leq n$ where $\sigma \in \Sigma_{n}$. Then the symmetrization of an element $x \in H^{*} D_{n}(R)$ is the sum without repetitions of all the elements $x^{\sigma}$ with $\sigma \in \Sigma_{n}$, i.e., the sum $\sum x^{\sigma}$ over the orbit of $x$.

2.1. Etale models. Before sketching the proof of Theorem 1.1 and its corollary we review some ingredients. Let $n$ be a nonnegative integer, $p$ a prime, and $R_{0}=$ $\mathbb{Z}[1 / p]$. Then the étale model $X_{n}(S)$ at $p$ is a topological space naturally assigned to each Noetherian $R_{0}$-algebra $S$ and the map

$$
f_{n}: B G L_{n}(S) \rightarrow X_{n}(S)
$$

is a natural transformation [4].

Proposition 2.4 ([1, 2.3], [5, 2.8, 2.11]). The following properties hold for any prime $p$ and nonnegative integer $n$ :

(1) If $S$ is a complete local ring with residue field $k$ of characteristic different from $p$, then the induced map $X_{n}(S) \rightarrow X_{n}(k)$ is a p-equivalence.

(2) If $S$ is a finite field of order prime to $p$, then $f_{n}$ is a $p$-equivalence.

(3) If $S$ is the field of complex numbers, then $X_{n}(S)$ is p-equivalent to the classifying space $B G L_{n}^{\text {top }}(S)$ of the Lie group $G L_{n}^{\text {top }}(S)$.

We recall that the cohomology ring of $B G L_{n}^{t o p}(\mathbb{C})$, the infinite complex Grassmannian, is a polynomial ring in the universal Chern classes $c_{1}, c_{2}, \ldots, c_{n}$. Also, if $\mathfrak{q}_{i}$ is a prime ideal of $R$ with the residue field $k_{i}$ of order $\equiv 1 \bmod p$ and $\mathfrak{q}_{\mathfrak{i}}$-adic completion $\hat{R}_{\mathfrak{q}_{\mathfrak{i}}}$, then the cohomology ring of $B G L_{n}\left(k_{i}\right)$ was calculated by Quillen in [8]. As a corollary of these known facts and Proposition 2.4 we have:

$$
H^{*} X_{n}\left(\hat{R}_{\mathfrak{q}_{\mathfrak{i}}}\right) \approx H^{*} B G L_{n}\left(k_{i}\right) \approx \mathbb{F}_{p}\left[c_{1}, c_{2}, \ldots, c_{n}\right] \otimes \Lambda\left(e_{i, 1}, e_{i, 2}, \ldots, e_{i, n}\right)
$$

where $\left|c_{j}\right|=2 j$ and $\left|e_{i, j}\right|=2 j-1$ for $1 \leq j \leq n$.

2.2. Proofs. Now we are ready to sketch the proofs of Theorem 2.1 and Corollary 2.2 .

Proof of Theorem [2.1] According to [2] there are $r=(p+1) / 2$ prime ideals of $R$, say $\mathfrak{q}_{i}$ for $1 \leq i \leq r$, and a commutative diagram in the category of Noetherian 
$R_{0}$-algebras

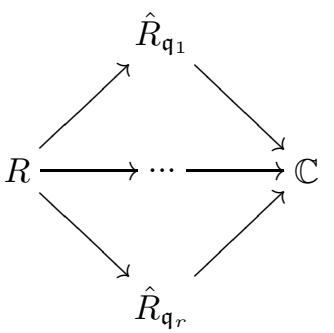

such that $X_{n}(\underline{(2.3)})$ is a homotopy cartesian diagram in the category of topological spaces. Moreover, the residue field of $\mathfrak{q}_{i}$, say $k_{i}$, has order $\equiv 1 \bmod p$ for $1 \leq i \leq r$. In particular, the formula (2.2) holds for all $1 \leq i \leq r$ and combining these formulae with (2.1), we deduce that the Eilenberg-Moore spectral sequence associated with the homotopy cartesian diagram $X_{n}([\sqrt{(2.3}))$ collapses to a ring isomorphism

$$
H^{*} X_{n}(R) \approx \bigotimes_{i=1}^{r} H^{*} X_{n}\left(\hat{R}_{\mathfrak{q}_{i}}\right) .
$$

Here the tensor product is over $H^{*} X_{n}(\mathbb{C})$ and hence the theorem follows from (2.2) and (2.1).

Proof of Corollary 2.2. By Dirichlet's Unit Theorem, $D_{1}(R)$ is a product of $r-1$ copies of the integers $\mathbb{Z}$ where $r=(p+1) / 2$ and a finite cyclic group $C$ with its $p$-primary part generated by the primitive $p$-th root of unity $\zeta$. By the Künneth theorem, it follows that $H^{*} B D_{n}(R)$ is a tensor product of $n(r-1)$ copies of $H^{*}(\mathbb{Z})$ and $n$ copies of $H^{*}(C)$ and therefore has the desired ring structure by standard calculations.

In order to prove the second part of the corollary, we can apply the same method as in the proof of Theorem 2.1. With the same notation, the restriction maps

$$
H^{*} B G L_{n}\left(k_{i}\right) \rightarrow H^{*} B D_{n}\left(k_{i}\right), \quad 1 \leq i \leq r
$$

can be identified according to [8, p. 564] with the symmetrization maps

$$
s_{i}: \mathbb{F}_{p}\left[c_{1}, \ldots, c_{n}\right] \otimes \Lambda\left(e_{i, 1}, \ldots, e_{i, n}\right) \rightarrow \mathbb{F}_{p}\left[x_{1}, \ldots, x_{n}\right] \otimes \Lambda\left(y_{i, 1}, \ldots, y_{i, n}\right) .
$$

Let us take the tensor product of all $s_{i}, 1 \leq i \leq r$, with respect to $\mathbb{F}_{p}\left[c_{1}, c_{2}, \ldots, c_{n}\right]$ in the source and $\mathbb{F}_{p}\left[x_{1}, x_{2}, \ldots, x_{n}\right]$ in the target. Then we can identify the source of $\bigotimes_{i=1}^{r} s_{i}$ with $H^{*} X_{n}(R)$ by Theorem 2.1, the target with $H^{*} B D_{n}(R)$ by the first part of the corollary, and hence, the map $\bigotimes_{i=1}^{r} s_{i}$ itself with $r e s_{n} \circ f_{n}^{*}$ by naturality. The injectivity of $\bigotimes_{i=1}^{r} s_{i}$ can be shown as in [8, Lemma 9].

\section{AN INVARIANT PROBLEM}

In this section, we deduce Theorem 1.1 from a more general invariant problem, which we formulate and solve after some preliminary steps.

3.1. Preliminary steps. In order to prove Theorem 1.1 we need to use the matrix block-multiplication $G L_{m}(R) \times G L_{n}(R) \rightarrow G L_{m+n}(R)$ for $m \geq 0$ and $n \geq 0$ (see 
Remark 3.5). This multiplication induces a mod $p$ homology ring structure on each of the direct sums appearing in the following diagram (compare to [3]):

$$
\bigoplus_{n=0}^{\infty} H_{*} B D_{n}(R) \stackrel{\oplus r e s_{n *}}{\longrightarrow} \bigoplus_{n=0}^{\infty} H_{*} B G L_{n}(R) \stackrel{\oplus f_{n *}}{\longrightarrow} \bigoplus_{n=0}^{\infty} H_{*} X_{n}(R)
$$

where $r e s_{n *}$ is dual to $r e s_{n}$ and $f_{n *}$ is dual to $f_{n}^{*}$ for $n \geq 0$ (the homology and cohomology are dual as vector spaces over $\mathbb{F}_{p}$ ). With respect to this ring structure the maps $\bigoplus_{n=0}^{\infty} r e s_{n *}$ and $\bigoplus_{n=0}^{\infty} f_{n *}$ are ring homomorphisms. This diagram induces a diagram of rings and ring homomorphisms

$$
\bigoplus_{n=0}^{\infty} H_{*} B D_{n}(R) \stackrel{\rho}{\rightarrow} \bigoplus_{n=0}^{\infty} I_{n}^{\prime} \rightarrow \bigoplus_{n=0}^{\infty} M_{n}^{\prime}
$$

where $V^{\prime}$ denotes the degreewise dual of the graded vector space $V$ over $\mathbb{F}_{p}$ and the maps in the diagram are direct sums of maps dual to the inclusions $M_{n} \subseteq I_{n}$ and $I_{n} \subseteq H^{*} B D_{n}(R)$ for $n \geq 0$ (same notation as in Theorem 1.1). These properties suggest an invariant problem, which we formulate in the next subsection.

3.2. Formulating the invariant problem. Let $r$ be a nonnegative integer and $F$ a field of characteristic different from 2. Both $r$ and $F$ are fixed throughout the discussion. For each $n \geq 0$ we define the following graded algebra over $F$ :

$$
A_{n}=F\left[x_{1}, x_{2}, \ldots, x_{n}\right] \otimes \bigotimes_{i=1}^{r} \Lambda\left(y_{i, 1}, y_{i, 2}, \ldots, y_{i, n}\right)
$$

with $\left|x_{j}\right|=2$ and $\left|y_{i, j}\right|=1$ for $1 \leq i \leq r$ and $1 \leq j \leq n$. The symmetric group $\Sigma_{n}$ acts on $A_{n}$ as described in Remark 2.3. With respect to this action we define the following subring of invariants:

$$
E_{n}=F\left[c_{1}, c_{2}, \ldots, c_{n}\right] \otimes \bigotimes_{i=1}^{r} \Lambda\left(e_{i, 1}, e_{i, 2}, \ldots, e_{i, n}\right)
$$

where $c_{j}$ and $e_{i, j}$ are the symmetrizations of $x_{1} \ldots x_{j}$ and $x_{1} \ldots x_{j-1} y_{i, j}$ for $1 \leq j \leq n$ and $1 \leq i \leq r$.

Let $V^{\prime}$ denote the degreewise dual of the graded vector space $V$ over $F$ and define an algebra structure on the direct sum $\bigoplus_{n=0}^{\infty} A_{n}^{\prime}$ as follows. For each $n \geq 0$, let $S(n)$ be the set of all sequences

$$
I=\left(k_{1}, \ldots, k_{n} ; \epsilon_{1,1}, \ldots, \epsilon_{1, n} ; \ldots ; \epsilon_{r, 1}, \ldots, \epsilon_{r, n}\right)
$$

of nonnegative integers with $\epsilon_{i, j} \in\{0,1\}$ for $1 \leq i \leq r$ and $1 \leq j \leq n$. The monomials

$$
x^{I}=x_{1}^{k_{1}} \ldots x_{n}^{k_{n}} \prod_{i=1}^{r} y_{i, 1}^{\epsilon_{i, 1}} \ldots y_{i, n}^{\epsilon_{i, n}}
$$

with $I \in S(n)$ as in (3.4) form an additive basis for $A_{n}$; let $\left(u_{I}\right)$ be the dual basis in $A_{n}^{\prime}$.

Definition 3.1. If $I \in S(n)$ is of the form (3.4) and $J \in S(m)$ is of the form

$$
J=\left(l_{1}, \ldots, l_{m} ; \phi_{1,1}, \ldots, \phi_{1, m} ; \ldots ; \phi_{r, 1}, \ldots, \phi_{r, m}\right),
$$

then we define the concatenation $I J \in S(n+m)$ by:

$$
I J=\left(k_{1}, \ldots, k_{n}, l_{1}, \ldots, l_{m} ; \epsilon_{1,1}, \ldots, \epsilon_{1, n}, \phi_{1,1}, \ldots, \phi_{1, m} ; \ldots ; \epsilon_{r, 1}, \ldots, \epsilon_{r, n}, \phi_{r, 1}, \ldots, \phi_{r, m}\right) .
$$


Definition 3.2. We define the product of any two basis elements $u_{I}$ and $u_{J}$ by the following equations:

$$
u_{I} u_{J}=\epsilon u_{I J}, \quad x^{I J}=\epsilon x^{I O} x^{O J}
$$

where $I \in S(n), J \in S(m), O$ stands for sequences of the form $(0,0, \ldots, 0)$ such that both $I O$ and $O J$ belong to $S(n+m)$, and $\epsilon \in\{-1,1\}$ is chosen such that the second equation is an identity in $A_{n+m}$.

Lemma 3.3. With respect to the product defined in 3.2. the direct sum $\bigoplus_{n=0}^{\infty} A_{n}^{\prime}$ is an algebra isomorphic to the tensor algebra generated by $A_{1}^{\prime}$.

Proof. Let $\theta$ be the linear map from the tensor algebra generated by $A_{1}^{\prime}$ which sends each basis element of the form $u_{I_{1}} \otimes u_{I_{2}} \otimes \ldots \otimes u_{I_{n}}$ to $\epsilon u_{I}$ where $n \geq 0, I_{k} \in S(1)$ for $1 \leq k \leq n, I=I_{1} I_{2} \ldots I_{n}$, and $\epsilon \in\{-1,1\}$ is chosen such that the following equation

$$
x^{I}=\epsilon x^{I_{1} O \ldots O} x^{O I_{2} O \ldots O} x^{O \ldots O I_{n}}
$$

is an identity in $A_{n}$. Here $O \in S(1)$ is a sequence of the form $(0,0, \ldots, 0)$ occurring $n-1$ times in each exponent. Then $\theta$ is the desired algebra isomorphism.

Now we can formulate the following invariant problem:

Theorem 3.4. For each $n \geq 0$, let $V_{n}$ be a graded linear subspace of $A_{n}$ that contains $E_{n}$ and assume that there is a ring structure on the direct sum $\bigoplus_{n=0}^{\infty} V_{n}^{\prime}$ such that the linear map

$$
g=\bigoplus_{n=0}^{\infty} g_{n}: \bigoplus_{n=0}^{\infty} A_{n}^{\prime} \rightarrow \bigoplus_{n=0}^{\infty} V_{n}^{\prime}
$$

is a ring homomorphism with respect to Definition 3.2 where $g_{n}$ is dual to the inclusion $V_{n} \subseteq A_{n}$ for each $n \geq 0$. Then $E_{2}=V_{2}$ implies $E_{n}=V_{n}$ for all $n \geq 0$.

Remark 3.5. Let us drop the hypothesis that $g$ is a ring homomorphism and assume instead that $V_{n}$ is a subring of the invariants $A_{n}^{\Sigma_{n}}$ that contains $E_{n}$ and whose image with respect to the map which kills all generators $x_{j}$ and $y_{i, j}$ for $j>2$ (and keeps the others) becomes the subring $E_{2} \subseteq A_{2}$. It is a pleasant warm-up exercise to show that in case $r=0$ and $r=1$ the invariants (and therefore $V_{n}$ ) agree with the ring $E_{n}$. However, for $r>1$ the invariants are bigger and it is not true that $E_{n}=V_{n}$. For instance, let $n \geq 3$ and let $y_{n}$ be the symmetrization of the monomial $y_{1,1} y_{1,2} y_{1,3} y_{2,1} y_{2,2} y_{2,3}$ in $A_{n}$. Then the subring $V_{n}=E_{n}\left[y_{n}\right]$ generated by $E_{n}$ and $y_{n}$ is a counterexample.

3.3. The proof of Theorem 1.1. We assume the notation and hypotheses of 1.1 By 2.1 and 2.2 the rings $H^{*} B D_{n}(R)$ and $M_{n}$ can be identified respectively with the rings $A_{n}$ and $E_{n}$ as defined in (3.2) and (3.3) for $F=\mathbb{F}_{p}, r=(p+1) / 2$, and $n \geq 0$. With these identifications, $I_{n}$ is a graded linear subspace of $A_{n}$ that contains $E_{n}$ for $n \geq 0$. Therefore, the map $\rho$ from (3.1) can be identified with the map $g$ from Theorem 3.4 for $V_{n}=I_{n}$. According to our discussion in \$3.1, the map $\rho$ is a ring homomorphism with respect to the algebra structure on $\bigoplus_{n=0}^{\infty} A_{n}^{\prime}$ induced from the matrix block-multiplication. By the Künneth theorem on homology, this algebra structure agrees with the algebra structure defined in 3.2 (compare to Lemma 3.3). Hence, Theorem 1.1 follows from Theorem 3.4. 
3.4. The proof of Theorem 3.4. We assume now the notation of $₫ 3.2$ Let us compose the map $g$ with the linear map

$$
h=\bigoplus_{n=0}^{\infty} h_{n}: \bigoplus_{n=0}^{\infty} V_{n}^{\prime} \rightarrow \bigoplus_{n=0}^{\infty} E_{n}^{\prime}
$$

where $h_{n}$ is dual to the inclusion $E_{n} \subseteq V_{n}$ for each $n \geq 0$. Let $t=h g$ and $t_{n}=h_{n} g_{n}$ for $n \geq 0$. Then we show that

Proposition 3.6. $\operatorname{ker}(t)$ is an ideal generated by $\operatorname{ker}\left(t_{2}\right)$.

Assuming this proposition and that $g$ is a ring homomorphism, then $\operatorname{ker}(g)$ is an ideal contained in $\operatorname{ker}(t)$ and hence, $E_{2}=V_{2}$ will imply that $\operatorname{ker}(g)=\operatorname{ker}(t)$. Because $g$ and $t$ are epimorphisms as direct sums of maps dual to inclusions, it follows that $h$ is an isomorphism. Therefore, $E_{n}=V_{n}$ for all $n \geq 0$.

Remark 3.7. A particular case of Proposition 3.6 appeared as [1, Lemma 3.3] but the proof given in [1, p.12] is obviously inaccurate.

3.5. The proof of Proposition 3.6. The strategy we are pursuing in proving 3.6 is the following. We first study the map $t$ in terms of additive bases and show that $\operatorname{ker}(t)$ is an ideal. Then we define a map $\alpha: S(n) \rightarrow S(n)$ which controls the way $t$ maps basis elements. By using $\alpha$, we show that if $\operatorname{ker}(t)$ is not generated by $\operatorname{ker}\left(t_{2}\right)$, then we have an infinite ascent phenomenon inside a certain subset $S$ of $S(n)$. Because $S$ will be finite, our assumption is false, proving Proposition 3.6.

Let us start with an additive basis for $E_{n}$ given by the monomials

$$
c^{I}=c_{1}^{k_{1}} \ldots c_{n}^{k_{n}} \prod_{i=1}^{r} e_{i, 1}^{\epsilon_{i, 1}} \ldots e_{i, n}^{\epsilon_{i, n}}
$$

where $I \in S(n)$ is of the form (3.4) and let $\left(v_{I}\right)$ be the dual basis in $E_{n}^{\prime}$. Then we have the following duality equations:

$$
\begin{aligned}
c^{K} & =\sum_{I \in S(n)}[K: I] x^{I} \text { for } K \in S(n), \\
t\left(u_{I}\right) & =\sum_{K \in S(n)}[K: I] v_{K} \text { for } I \in S(n)
\end{aligned}
$$

where the coefficients $[K: I] \in F$ are zero almost everywhere in each sum.

Lemma 3.8. There is a ring structure on the direct sum $\bigoplus_{n=0}^{\infty} E_{n}^{\prime}$ such that $t$ is a ring homomorphism with respect to Definition 3.2

Proof. Let us define a ring structure on the direct sum $\bigoplus_{n=0}^{\infty} E_{n}^{\prime}$ by the following equations which are similar to 3.2

$$
v_{K} v_{L}=\delta v_{K L}, \quad c^{K L}=\delta c^{K O} c^{O L}
$$

where $K \in S(n), L \in S(m)$, and $\delta \in\{-1,1\}$ is chosen such that the second equation is an identity in $E_{n+m}$. Then we can use (3.5), (3.6), and 3.2 to show that $t$ is a ring homomorphism.

Next, we need a way to bound indices $I$ by natural numbers (see 3.9) . Also, we use the following lexicographical order: two sequences of integers $a=\left(a_{1}, \ldots, a_{m}\right)$ and $a^{\prime}=\left(a_{1}^{\prime}, \ldots, a_{m}^{\prime}\right)$ of the same length $m \geq 0$ are in the relation $a>a^{\prime}$ if $a_{1}>a_{1}^{\prime}$ or there is $1 \leq s<m$ such that $a_{j}=a_{j}^{\prime}$ for $1 \leq j \leq s$ and $a_{s+1}>a_{s+1}^{\prime}$. 
Definition 3.9. Let $n \geq 0$ and let $I \in S(n)$ be of the form (3.4). Then we define the degree of $I$ by

$$
\operatorname{deg}(I)=\sum_{j=1}^{n}\left(k_{j}+\sum_{i=1}^{r} \epsilon_{i, j}\right) .
$$

Lemma 3.10. Let $n \geq 0$. Then there is an injective map $\alpha: S(n) \rightarrow S(n)$ such that for any $I \in S(n)$ and $K \in S(n)$ the following conditions hold:

(1) $[K: I]=1$ if $I=\alpha(K)$.

(2) $[K: I]=0$ if $I>\alpha(K)$.

(3) If $I$ is of the form (3.4), then $I$ is in the image of $\alpha$ if and only if

$$
k_{j}-k_{j+1} \geq \sum_{i=1}^{r} \epsilon_{i, j+1} \text { for all } 1 \leq j<n .
$$

(4) $[K: I]=0$ if $\operatorname{deg}(I) \neq \operatorname{deg}(\alpha(K))$.

Proof. Let us assume that $I \in S(n)$ has the form (3.4) and $K$ the form

$$
K=\left(a_{1}, \ldots, a_{n} ; \phi_{1,1}, \ldots, \phi_{1, n} ; \ldots ; \phi_{r, 1}, \ldots, \phi_{r, n}\right) .
$$

Then $x^{I}$ is lexicographically with respect to $I$ the leading term of the expansion of $c^{K}$ as in (3.5) if and only if:

$$
\begin{aligned}
k_{j} & =a_{j}+\ldots+a_{n}+\sum_{i=1}^{r} \phi_{i, j+1}+\ldots+\sum_{i=1}^{r} \phi_{i, n} \quad \text { for } 1 \leq j<n, \\
k_{n} & =a_{n}, \quad \text { for } 1 \leq j \leq n \text { and } 1 \leq i \leq r .
\end{aligned}
$$

Given $I \in S(n)$, this system of equations has a solution $K \in S(n)$ if and only if $I$ satisfies the condition (3) of the lemma. Moreover, if this condition is satisfied, then the solution $K$ is unique. Hence, if we define $\alpha(K)$ to be the only element in $S(n)$ such that $x^{\alpha(K)}$ is the leading term of $c^{K}$, then the map $\alpha$ is injective and satisfies (1) to (3). In order to prove (4) we only need to observe that all monomials $x^{I}$ occurring with nonzero coefficients in the expansion of $c^{K}$ have the same value for $\operatorname{deg}(I)$ and $x^{\alpha(K)}$ is one of these monomials.

With these preparations and notation, we can prove Proposition 3.6 as follows. By Lemma 3.8, $t$ is a ring homomorphism and therefore, $\operatorname{ker}(t)$ contains the ideal generated by $\operatorname{ker}\left(t_{2}\right)$, denoted $\left(\operatorname{ker}\left(t_{2}\right)\right)$. Now, assume that $\operatorname{ker}(t)$ is bigger than $\left(\operatorname{ker}\left(t_{2}\right)\right)$. For each $n \geq 0$ and $d \geq 0$, let $M$ be the set of all elements $x \in A_{n}^{\prime}$ such that $x \in \operatorname{ker}(t), x \notin\left(\operatorname{ker}\left(t_{2}\right)\right)$, and $x$ can be written in the form

$$
x=\sum_{I \in S(n), \operatorname{deg}(I)=d}(x: I) u_{I}
$$

where we define $(x: I) \in F$ for all $I \in S(n)$ by setting $(x: I)=0$ if $\operatorname{deg}(I) \neq d$. Let us fix $n$ and $d$ such that the set $M$ is not empty (we can do this according to our assumption, (3.6), and Lemma [3.10 (4)). Now, let $S$ be the set of all $I \in S(n)$ satisfying the following conditions: $\operatorname{deg}(I)=d$ and there is $x \in M$ such that $(x: J)=0$ for all $J<I$. This set is finite and not empty. Let $I_{0} \in S$ be lexicographically the maximal element of $S$.

We claim that we can find an element $I_{0}^{\prime} \in S$ which contradicts the maximality property of $I_{0}$. More specifically, we have the following 
Proposition 3.11. Let $x_{0} \in M$ such that $\left(x_{0}: I\right)=0$ for all $I<I_{0}$. Then there is $u \in\left(\operatorname{ker}\left(t_{2}\right)\right)$ such that $u$ can be written in the form (3.7) with $\left(u: I_{0}\right)=\left(x_{0}: I_{0}\right)$ and $(u: I)=0$ for $I<I_{0}$.

Now, the element $x_{0}^{\prime}=x_{0}-u$ belongs to $M$ and there is $I_{0}^{\prime} \in S(n)$ such that $\operatorname{deg}\left(I_{0}^{\prime}\right)=d$ and $\left(x_{0}^{\prime}: I\right)=0$ for all $I<I_{0}^{\prime}$ but $\left(x_{0}^{\prime}: I_{0}^{\prime}\right) \neq 0$. Therefore, $I_{0}^{\prime} \in S$ and by the properties of $u$ and $x_{0},\left(x_{0}^{\prime}: I\right)=0$ for all $I \leq I_{0}$. It follows that $I_{0}^{\prime}>I_{0}$, proving the claim and Proposition 3.6 .

3.6. The proof of Proposition 3.11. We need to make use of the map $\alpha$ and its properties given in Lemma 3.10 .

Case 1: Assume that there is $K \in S(n)$ such that $I_{0}=\alpha(K)$. By [3.10 (1) (2) we have $\left[K: I_{0}\right]=1$ and $[K: I]=0$ for all $I>I_{0}$. Combining these with (B.6) and the properties of $x_{0}$ and $I_{0}$, we have

$$
0=\left\langle c^{K}, t\left(x_{0}\right)\right\rangle=\sum_{I \in S(n), \operatorname{deg}(I)=d}[K: I]\left(x_{0}: I\right)=\left(x_{0}: I_{0}\right)
$$

where $\langle$,$\rangle is the duality pairing. Hence, u=0$ has the desired properties.

Case 2: Assume that there is no $K \in S(n)$ such that $I_{0}=\alpha(K)$. Then by 3.10 (3) there is $1 \leq j<n$ such that $I_{0}=I_{1} I_{2} I_{3}$ where $I_{1} \in S(j-1), I_{2} \in S(2)$, and $I_{3} \in S(n-j-1)$ are of the form

$$
\begin{aligned}
& I_{1}=\left(k_{1}, \ldots, k_{j-1} ; \epsilon_{1,1}, \ldots, \epsilon_{1, j-1} ; \ldots ; \epsilon_{r, 1}, \ldots, \epsilon_{r, j-1}\right), \\
& I_{2}=\left(k_{j}, k_{j+1} ; \epsilon_{1, j}, \epsilon_{1, j+1} ; \ldots ; \epsilon_{r, j}, \epsilon_{r, j+1}\right) \text { with } k_{j}-k_{j+1}<\sum_{i=1}^{r} \epsilon_{i, j+1}, \\
& I_{3}=\left(k_{j+2}, \ldots, k_{n} ; \epsilon_{1, j+2}, \ldots, \epsilon_{1, n} ; \ldots ; \epsilon_{r, j+2}, \ldots, \epsilon_{r, n}\right) .
\end{aligned}
$$

Subcase 2(a): Assume that $t\left(u_{I_{2}}\right)=0$. Then $u_{I_{0}}= \pm u_{I_{1}} u_{I_{2}} u_{I_{3}} \in\left(\operatorname{ker}\left(t_{2}\right)\right)$ and $u=\left(x_{0}: I_{0}\right) u_{I_{0}}$ has the desired properties.

Subcase 2(b): Assume that $t\left(u_{I_{2}}\right) \neq 0$. Let $d^{\prime}=\operatorname{deg}\left(I_{2}\right)$ and define $T$ to be the set of all $K \in S(2)$ such that $\operatorname{deg}(\alpha(K))=d^{\prime}$. Because $\alpha$ is injective, $T$ is a set of finite order, say $m$. Let us label the elements of $T$ by $K_{1}, K_{2}, \ldots, K_{m}$ such that $\alpha\left(K_{1}\right)<\alpha\left(K_{2}\right)<\ldots<\alpha\left(K_{m}\right)$.

Lemma 3.12. There is $1 \leq s \leq m$ such that $I_{2}<\alpha\left(K_{s}\right)$ and $\left[K_{i}: I_{2}\right]=0$ for all $1 \leq i<s$.

Proof. By (3.6) and 3.10 (4), we have

$$
t\left(u_{I_{2}}\right)=\sum_{i=1}^{m}\left[K_{i}: I_{2}\right] v_{K_{i}} .
$$

Because $t\left(u_{I_{2}}\right) \neq 0$, there is $1 \leq s \leq m$ such that $\left[K_{s}: I_{2}\right] \neq 0$ and $\left[K_{i}: I_{2}\right]=0$ for $1 \leq i<s$. By 3.10 (3) there is no $K \in S(2)$ such that $I_{2}=\alpha(K)$ and combining this with $3.10(2)$, we get $I_{2}<\alpha\left(K_{s}\right)$.

Corollary 3.13. With $s$ as in Lemma 3.12, the following system of equations

$$
0=\left[K_{j}: I_{2}\right]+\sum_{i=s}^{m}\left[K_{j}: \alpha\left(K_{i}\right)\right] \lambda_{i} \text { for all } 1 \leq j \leq m
$$

has a solution $\lambda_{s}, \lambda_{s+1}, \ldots, \lambda_{m} \in F$. 
Proof. By [3.10 (1) (2), we have $\left[K_{i}: \alpha\left(K_{i}\right)\right]=1$ for all $s \leq i \leq m$ and $\left[K_{j}\right.$ : $\left.\alpha\left(K_{i}\right)\right]=0$ for all $1 \leq j<i \leq m$. Combining these with [3.12, we deduce that the system has indeed a solution.

Let $\lambda_{i} \in F$ for $s \leq i \leq m$ be a solution of the system in Corollary 3.13 and define

$$
w=u_{I_{2}}+\sum_{i=s}^{m} \lambda_{i} u_{\alpha\left(K_{i}\right)} .
$$

Then we have

$$
\begin{aligned}
t(w) & =\sum_{j=1}^{m}\left(\left[K_{j}: I_{2}\right]+\sum_{i=s}^{m}\left[K_{j}: \alpha\left(K_{i}\right)\right] \lambda_{i}\right) v_{K_{j}} \quad \text { by (3.6) and 3.10 (4) } \\
& =0 \text { by 3.13 }
\end{aligned}
$$

Therefore, $w \in \operatorname{ker}\left(t_{2}\right)$ and $u= \pm\left(x_{0}: I_{0}\right) u_{I_{1}} w u_{I_{3}}$ has the desired properties, proving Proposition 3.11 .

\section{Etale obstruction Classes}

In this section we keep the hypotheses and notation from $\$ 2$ and show that the condition $M_{2}=I_{2}$ in Theorem [1.1 is satisfied if certain homology classes vanish.

Namely, let $S L_{2}(R)$ be the special linear group of $2 \times 2$ matrices over $R$ with determinant 1 and consider the following map:

$$
\iota: D_{1}(R) \rightarrow S L_{2}(R), \quad \iota(a)=\left(\begin{array}{cc}
a^{-1} & 0 \\
0 & a
\end{array}\right) \text { for } a \in D_{1}(R) .
$$

By Corollary 2.2 the mod $p$ group cohomology $H^{*} D_{1}(R)$ can be identified with the ring

$$
\mathbb{F}_{p}[x] \otimes \bigotimes_{i=1}^{r} \Lambda\left(y_{i}\right), r=\frac{p+1}{2},|x|=2,\left|y_{i}\right|=1 \text { for } 1 \leq i \leq r .
$$

For each $I \in S(1)$ of the form $I=\left(k, \epsilon_{1}, \epsilon_{2}, \ldots, \epsilon_{r}\right)$ as in $\$ 3.2$ let

$$
x^{I}=x^{k} \prod_{i=1}^{r} y_{i}^{\epsilon_{i}} \quad \text { and } \quad a(I)=\frac{1}{2}\left(\epsilon_{1}+\epsilon_{2}+\ldots+\epsilon_{r}-k\right)
$$

where we recall that $\epsilon_{i} \in\{0,1\}$ for $1 \leq i \leq r$. Then $H^{*} D_{1}(R)$ has an additive basis given by the monomials $x^{I}$ with $I \in S(1)$. Let $\left(u_{I}\right)$ be the dual basis in $H_{*} D_{1}(R)$. Then the map $\iota_{*}$ induced by $\iota$ on $\bmod p$ homology sends each basis element $u_{I}$ with $I \in S(1)$ to a homology class $\iota_{*}\left(u_{I}\right)$ in $H_{*} S L_{2}(R)$.

Definition 4.1. Let $\beta$ be the Bockstein derivation on $\bmod p$ cohomology. We call an identification of $H^{*} D_{1}(R)$ with the ring (4.2) admissible if $x \in \beta H^{1} D_{1}(R)$.

Definition 4.2. Given an admissible identification, we say that $\iota_{*}\left(u_{I}\right) \in H_{*} S L_{2}(R)$ where $I \in S(1)$ is an étale obstruction class if $a(I)$ is a positive integer.

Proposition 4.3. Let $p$ be an odd regular prime and fix an admissible identification of $H^{*} D_{1}(R)$ with (4.2). Then the linear subspace of $H_{*} S L_{2}(R)$ spanned by the étale obstruction classes is trivial if and only if the condition $M_{2}=I_{2}$ in Theorem 1.1 is satisfied. 
Remark 4.4. For $p=3$, there is only one sequence $I \in S(1)$ satisfying 4.2 namely $I=(0,1,1)$. Therefore, given an admissible identification, there is only one obstruction class living in $\mathrm{H}_{2} S L_{2}(R)$. By [1, (5.1)], this homology group is zero, reproving 1.2 as a consequence of 1.1 and 4.3 For $p>3$, it is an open problem whether $M_{2}=I_{2}$ or not.

4.1. The proof of Proposition 4.3. The idea is to use the following commutative diagram of groups and group homomorphisms:

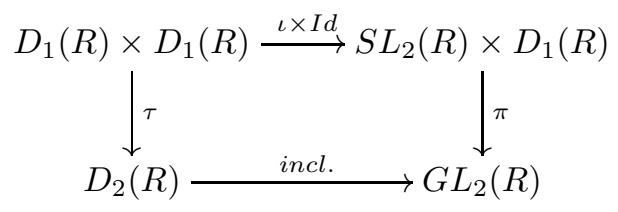

where $\iota$ is the map (4.1), Id is the identity map of $D_{1}(R)$, incl. is the inclusion map $D_{2}(R) \subset G L_{2}(R)$, and the other two maps are defined by the formulae

$$
\begin{aligned}
\tau(a, b) & =\iota(a)\left(\begin{array}{ll}
b & 0 \\
0 & b
\end{array}\right), \\
\pi(A, b) & =A\left(\begin{array}{ll}
b & 0 \\
0 & b
\end{array}\right)
\end{aligned}
$$

for $a, b \in D_{1}(R)$ and $A \in S L_{2}(R)$ (on the right-hand side we use matrix multiplication). This diagram induces a commutative diagram on mod $p$ cohomology

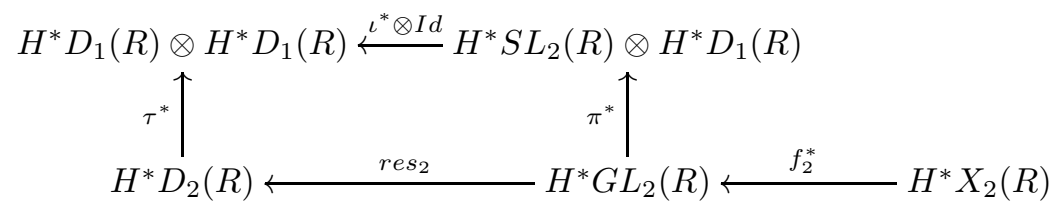

where we have identified $H^{*} G L_{2}(R)$ with $H^{*} B G L_{2}(R)$. According to Theorem 2.1 Corollary 2.2, and their proofs, there are identifications

$$
\begin{aligned}
& H^{*} D_{1}(R) \stackrel{\theta_{0}}{\approx} \mathbb{F}_{p}[x] \otimes \bigotimes_{i=1}^{r} \Lambda\left(y_{i}\right), \\
& H^{*} D_{2}(R) \approx \mathbb{F}_{p}\left[x_{1}, x_{2}\right] \otimes \bigotimes_{i=1}^{r} \Lambda\left(y_{i, 1}, y_{i, 2}\right), \\
& H^{*} X_{2}(R) \approx \mathbb{F}_{p}\left[c_{1}, c_{2}\right] \otimes \bigotimes_{i=1}^{r} \Lambda\left(e_{i, 1}, e_{i, 2}\right)
\end{aligned}
$$

such that $x=\beta\left(y_{i}\right), x_{1}=\beta\left(y_{i, 1}\right), x_{2}=\beta\left(y_{i, 2}\right), c_{1}=\beta\left(e_{i, 1}\right), 2 c_{2}=\beta\left(e_{i, 2}\right), r e s_{2} \circ f_{2}^{*}$ is the inclusion determined by $e_{i, 1}=y_{i, 1}+y_{i, 2}, e_{i, 2}=x_{1} y_{i, 2}+x_{2} y_{i, 1}$, and $\tau^{*}$ is the map determined by $\tau^{*}\left(y_{i, 1}\right)=1 \otimes y_{i}-y_{i} \otimes 1, \tau^{*}\left(y_{i, 2}\right)=y_{i} \otimes 1+1 \otimes y_{i}$ for all $1 \leq i \leq r$. Assuming these identifications and notation, we have the following lemmas which imply Proposition 4.3 . 
Lemma 4.5. The map $\tau^{*}$ is a ring isomorphism mapping the image $M_{2}$ of res $_{2} \circ f_{2}^{*}$ to the following subring inside $H^{*} D_{1}(R) \otimes H^{*} D_{1}(R)$ :

$$
\tau^{*}\left(M_{2}\right)=\mathbb{F}_{p}\left[x^{2} \otimes 1,1 \otimes x\right] \otimes \bigotimes_{i=1}^{r} \Lambda\left(x y_{i} \otimes 1,1 \otimes y_{i}\right) .
$$

Proof. By applying $\beta$ to $\tau^{*}\left(y_{i, 1}\right)$ and $\tau^{*}\left(y_{i, 2}\right)$ for any $1 \leq i \leq r$, we obtain $\tau^{*}\left(x_{1}\right)=$ $1 \otimes x-x \otimes 1$ and $\tau^{*}\left(x_{2}\right)=1 \otimes x+x \otimes 1$. Hence, we easily see that $\tau^{*}$ is surjective and therefore, an isomorphism, because 2 is invertible ( $p$ is odd) and the source and the target of $\tau^{*}$ are graded vector spaces of the same finite dimension in each degree. Also, $\tau^{*}\left(e_{i, 1}\right)=2\left(1 \otimes y_{i}\right), \tau^{*}\left(e_{i, 2}\right)=2\left(1 \otimes x y_{i}-x y_{i} \otimes 1\right)$ for $1 \leq i \leq r$ and by applying $\beta, \tau^{*}\left(c_{1}\right)=2(1 \otimes x), \tau^{*}\left(c_{2}\right)=2\left(1 \otimes x^{2}-x^{2} \otimes 1\right)$. Therefore, $1 \otimes y_{i}$, $1 \otimes x, x y_{i} \otimes 1$, and $x^{2} \otimes 1$ for $1 \leq i \leq r$ generate $\tau^{*}\left(M_{2}\right)$ as a ring.

Lemma 4.6. The image of $\iota^{*}$ is invariant with respect to the ring automorphism of $H^{*} D_{1}(R)$ given by $x \mapsto-x, y \mapsto-y_{i}$ for $1 \leq i \leq r$.

Proof. The group automorphism of $D_{1}(R)$ given by $a \mapsto a^{-1}$ for $a \in D_{1}(R)$ induces the multiplication by $(-1)$ map on $H^{1} D_{1}(R)$. The map $\iota(a) \rightarrow \iota\left(a^{-1}\right)$ for $a \in D_{1}(R)$ is a conjugation inside $S L_{2}(R)$ which induces the trivial map on $H^{*} S L_{2}(R)$. Hence, we conclude that the image of $\iota^{*}$ is invariant with respect to the map described in the lemma.

Lemma 4.7. If all étale obstruction classes associated to $\theta_{0}$ in $H_{*} S L_{2}(R)$ vanish, then $\tau^{*}\left(I_{2}\right)=\tau^{*}\left(M_{2}\right)$.

Proof. The idea is to use the additive basis of $H^{*} D_{1}(R) \otimes H^{*} D_{1}(R)$ given by the monomials $x^{I} \otimes x^{J}$ with $I, J \in S(1)$. By Lemma 4.5, the monomials $x^{I} \otimes x^{J}$ where $I, J \in S(1)$ with $a(I)$ a non-positive integer form an additive basis for $\tau^{*}\left(M_{2}\right)$. By Lemma 4.6, the monomials $x^{I} \otimes x^{J}$ where $I, J \in S(1)$ with $a(I)$ an integer span a linear subspace that contains the image of $\iota^{*} \otimes I d$. Finally, by our hypothesis, given any $z \in H^{*} S L_{2}(R), z^{\prime} \in H^{*} D_{1}(R)$, and $I, J \in S(1)$ with $a(I)$ a positive integer, the following equation is satisfied:

$$
\left\langle\iota^{*}(z) \otimes z^{\prime}, u_{I} \otimes u_{J}\right\rangle=\left\langle z, \iota_{*}\left(u_{I}\right)\right\rangle\left\langle z^{\prime}, u_{J}\right\rangle=0 .
$$

Here, $\left(u_{L}\right)$ denotes the dual basis in $H_{*} D_{1}(R)$ of the basis $\left(x^{L}\right)$ in $H^{*} D_{1}(R)$ where $L \in S(1)$ and $\langle$,$\rangle is the duality pairing. Hence, by putting together these obser-$ vations we conclude that $\operatorname{Im}\left(\iota^{*} \otimes I d\right) \subseteq \tau^{*}\left(M_{2}\right)$. According to the diagram (4.3), we have $\tau^{*}\left(M_{2}\right) \subseteq \tau^{*}\left(I_{2}\right) \subseteq \operatorname{Im}\left(\iota^{*} \otimes I d\right)$ and the conclusion follows.

Lemma 4.8. If there is an étale obstruction class associated to $\theta_{0}$ in $H_{*} S L_{2}(R)$ that is not zero, then $\tau^{*}\left(I_{2}\right) \neq \tau^{*}\left(M_{2}\right)$.

Proof. By a spectral sequence argument, $\operatorname{Im}\left(\pi^{*}\right)$ agrees with the invariants of $H^{*} S L_{2}(R) \otimes H^{*} D_{1}(R)$ with respect to the action of the cokernel of $\pi$, which is $D_{1}(R) / D_{1}(R)^{2} \approx(\mathbb{Z} / 2)^{r}$. Because this group acts trivially on $H^{*} D_{1}(R) \otimes H^{*} D_{1}(R)$ and 2 is invertible, we conclude that

$$
\operatorname{Im}\left(\iota^{*} \otimes I d\right)=\left(\iota^{*} \otimes I d\right)\left(\operatorname{Im}\left(\pi^{*}\right)\right)=\tau^{*}\left(I_{2}\right) .
$$

With the same notation as in the proof of Lemma 4.7 if there is $I_{0} \in S(1)$ with $a\left(I_{0}\right)$ a positive integer and $\iota_{*}\left(u_{I_{0}}\right) \neq 0$, then there is $z \in H^{*} S L_{2}(R)$ such that $\left\langle z, \iota_{*}\left(u_{I_{0}}\right)\right\rangle \neq 0$. Consequently, $\iota^{*}(z) \otimes 1$ belongs to $\tau^{*}\left(I_{2}\right)$ by (4.4) but not to $\tau^{*}\left(M_{2}\right)$ by Lemma 4.5. 
Lemma 4.9. The linear subspace of $H_{*} S L_{2}(R)$ spanned by the étale obstruction classes is independent of the admissible identification chosen.

Proof. Let $\left(x ; y_{1}, y_{2}, \ldots, y_{r}\right)$ and $\left(\bar{x} ; \bar{y}_{1}, \bar{y}_{2}, \ldots, \bar{y}_{r}\right)$ be two sets of ring generators for $H^{*} D_{1}(R)$ with $x, \bar{x} \in \beta H^{1} D_{1}(R)$ and $y_{i}, \bar{y}_{i} \in H^{1} D_{1}(R)$ for $1 \leq i \leq r$. Because $\beta H^{1} D_{1}(R)$ is a one-dimensional vector space, $\bar{x}=\lambda x$ for some nonzero scalar $\lambda \in \mathbb{F}_{p}$. Because $H^{1} D_{1}(R)$ is $r$-dimensional, there is $\left(\theta_{i, j}\right) \in G L_{r}\left(\mathbb{F}_{p}\right)$ such that

$$
\bar{y}_{i}=\sum_{j=1}^{r} \theta_{i, j} y_{j} \quad \text { for } 1 \leq i \leq r .
$$

Then, for each $I \in S(1)$ of the form $I=\left(k ; \epsilon_{1}, \epsilon_{2}, \ldots, \epsilon_{r}\right)$ the monomial

$$
\bar{x}^{I}=\bar{x}^{k} \prod_{i=1}^{r} \bar{y}_{i}^{\epsilon_{i}}
$$

can be written as a linear combination of monomials of the form $x^{I_{\sigma}}$ where $\sigma \in \Sigma_{r}$ and $I_{\sigma}=\left(k ; \epsilon_{\sigma(1)}, \epsilon_{\sigma(2)}, \ldots, \epsilon_{\sigma(r)}\right)$ and vice versa. Observe that $a(I)=a\left(I_{\sigma}\right)$ for any $I \in S(1)$ and $\sigma \in \Sigma_{r}$. Let $\left(u_{I}\right)$ be the dual basis of $\left(x^{I}\right)$ and $\left(\bar{u}_{I}\right)$ the dual of $\left(\bar{x}^{I}\right)$ where $I \in S(1)$. Hence, we conclude that each étale obstruction class $\iota_{*}\left(u_{J}\right)$ with $J \in S(1)$ is a linear combination of $\iota_{*}\left(\bar{u}_{I}\right)$ where $I \in S(1)$ with $a(I)$ a positive integer, and vice versa.

4.2. More general rings of integers. According to [2], we can generalize Theorem 1.1 as follows. Suppose that $F$ is a finite field extension of $\mathbb{Q}$ and that $\mathcal{O}_{F}$ is the ring of algebraic integers in $F$. Assume that $\mathcal{O}_{F}$ satisfies the following conditions:

(1) in $\mathcal{O}_{F}$ there is only one prime ideal above $p$;

(2) the Picard group of $\mathcal{O}_{F}$ has no $p$-torsion;

(3) $\mathcal{O}_{F}$ contains a primitive $p$-th root of unity.

Let $R=\mathcal{O}_{F}[1 / p]$ and $r=r_{2}+1$ where $r_{2}$ is half the number of complex embeddings of $F$. Then we can define the étale obstruction classes associated to an admissible identification of $H^{*} D_{1}(R)$ with a ring of the form (4.2) exactly as in Definition 4.2. With these adjustments, the proofs go through word by word and we have the following overall result:

Theorem 4.10. Let $R=\mathcal{O}_{F}[1 / p]$ with $p$ an odd prime and $\mathcal{O}_{F}$ a ring of algebraic integers satisfying (1) to (3) above. Then the linear subspace of $H_{*} S L_{2}(R)$ spanned by the étale obstruction classes associated to an admissible identification is trivial if and only if the maps res ${ }_{n}$ and $r e s_{n} \circ f_{n}^{*}$ as in Theorem 1.1 have the same image for all $n \geq 0$, including in the stable range.

Remark 4.11. The statement that $r e s_{n}$ and $r e s_{n} \circ f_{n}^{*}$ have the same image in the stable range was proved in a paper of S. Mitchell [7]. It should be noted that $H^{*} B D_{n}(R)$ and $H^{*} X_{n}(R)$ are completely stable in the sense that they are images of the $n=\infty$ versions, but this is not true for $H^{*} B G L_{n}(R)$. Hence the case $n<\infty$ does not follow from Mitchell's result.

\section{REFERENCES}

[1] Anton, M.F.: On a conjecture of Quillen at the prime 3, J. Pure Appl. Algebra 144 (1999), 1-20. MR 2000m:19003 
[2] Anton, M.F.: Etale approximations and the mod $\ell$ cohomology of $G L_{n}$, Cohomological methods in homotopy theory (Bellaterra, 1998), 1-10, Progr. Math., 196, Birkhäuser, Basel, 2001. MR 2002h:11119

[3] Dwyer, W.G.: Exotic cohomology for $G L_{n}\left(\mathbb{Z}\left[\frac{1}{2}\right]\right)$, Proc. Amer. Math. Soc. 126 (1998), 21592167. MR 2000a:57092

[4] Dwyer, W.G. and Friedlander, E.M.: Algebraic and etale K-theory, Trans. Amer. Math. Soc. 292 (1985), 247-280. MR 87h:18013

[5] Dwyer, W.G. and Friedlander, E.M.: Topological models for arithmetic, Topology 33 (1994), 1-24. MR 95h:19004

[6] Lichtenbaum, S.: Values of zeta-functions, étale cohomology, and algebraic K-theory. Algebraic $K$-theory, II: "Classical" algebraic $K$-theory and connections with arithmetic (Proc. Conf., Battelle Memorial Inst., Seattle, Wash., 1972), pp. 489-501. Lecture Notes in Math., Vol. 342, Springer, Berlin, 1973. MR [53:10765

[7] Mitchell, S.A.: Units and general linear group cohomology for a ring of algebraic integers, Math. Zeit. 228 (1998), 207-220. MR 99k:19002

[8] Quillen, D.: On the cohomolgy and K-theory of the general linear groups over a finite field, Annals of Math. 96 (1972), 552-586. MR 47:3565

[9] Voevodsky, V.: The Milnor conjecture, preprint MPI, 1997.

Department of Pure Mathematics, University of Sheffield, Hicks Building, Sheffield S3 7RH, United Kingdom and IMAR, P.O. Box 1-764, Bucharest, Romania 70700

Current address: Department of Mathematics, University of Kentucky, 715 POT, Lexington, Kentucky 40506-0027

E-mail address: Marian.Anton@imar.ro 